05

\title{
Сверхизлучение и синтез наночастиц металла в полистирольном композите с многоспиновыми комплексами Еu при быстром одноосном сдавливании
}

\author{
(C) А.И. Александров, ${ }^{1}$ В.Г. Шевченко, ${ }^{1}$ С.С. Абрамчук, ${ }^{2}$ С.Б. Зезин, ${ }^{3}$ С.В. Фокин, ${ }^{4}$ В.И. Овчаренко \\ ${ }^{1}$ Институт синтетических полимерных материалов им. Н.С. Ениколопова РАН, \\ 117393 Москва, Россия \\ ${ }^{2}$ Институт элементоорганических соединений им. А.Н. Несмеянова РАН, \\ 119991 Москва, Россия \\ ${ }^{3}$ Московский государственный университет им. М.В. Ломоносова, \\ 119991 Москва, Россия \\ ${ }^{4}$ Международный томографический центр СО РАН, \\ 630090 Новосибирск, Россия \\ e-mail: alivaleksandr@mail.ru
}

Поступило в Редакцию 25 июня 2021 г.

В окончательной редакции 27 октября 2021 г.

Принято к публикации 3 ноября 2021 г.

Синтезированы композиты гетероспинового молекулярного магнетика $\left[\mathrm{Eu}^{\mathrm{III}}(\mathrm{SQ})_{3}\right.$ bipy] в полистирольной $(\mathrm{PS})$ матрице. Комплекс $\left[\mathrm{Eu}^{\text {III }}(\mathrm{SQ})_{3}\right.$ bipy] содержит четыре парамагнитных центра - ион $\mathrm{Eu}^{3+}$ и три лиганда SQ (анион-радикалы SQ-3,6-ди-трет-бутилбензохинона); bipy (бипиридил) — диамагнитен. Установлено, что интенсивная механическая активация образцов $\left[\mathrm{Eu}^{\mathrm{III}}(\mathrm{SQ})_{3}\right.$ bipy]/PS приводит к реологическому взрыву, в результате которого наблюдается радиочастотное сверхизлучение, возникновение свободных электронов и образование металлических наночастиц Еu. Длительность данного процесса - $10 \mathrm{~ns}$.

Ключевые слова: излучение, импульс, упругая волна, европий.

DOI: $10.21883 /$ JTF.2022.02.52015.194-21

\section{Введение}

Эффект механической активации радиочастотного сверхизлучения (СИ) (эффект МАРСИ) был обнаружен в полимерных композитах, содержащих элементоорганические биядерные комплексы кобальта или марганца, которые проявляли эффект Дзялошинского-Мориа [1-5]. Механическая активация заключалась в воздействии импульса упругих волн от реологического взрыва исследуемого композита при быстром одноосном сжатии [1-3], или в параметрическом режиме при воздействии внешним реологическим взрывом, когда импульс упругих волн вводился в образец по стальному волноводу $[4,5]$. Представляет научный и практический интерес поиск новых классов твердофазных объектов, реализующих этот эффект, а также развитие новых методов обнаружения эффекта МАРСИ. Отметим, что явления СИ [6], реологического взрыва [7], эффект Дзялошинского-Мориа $[8,9]$ были обнаружены достаточно давно и привлекают внимание исследователей до настоящего времени.

Сравнительно недавно было установлено, что:

a) при реологическом взрыве полистирола (PS) в нем возникают свободные нанообъемы (нанополости) с подвижными стенками [10];

б) в композите $\left[\mathrm{Eu}^{\mathrm{III}}(\mathrm{SQ})_{3}\right.$ bipy $] / \mathrm{PS}$ при реологическом взрыве фиксируется радиочастотное сверхизлучение [11]; в) комплекс $\left[\mathrm{Eu}^{\mathrm{III}}(\mathrm{SQ})_{3}\right.$ bipy] является молекулярным магнетиком [12].

Здесь и далее $\left[\mathrm{Eu}^{\mathrm{III}}(\mathrm{SQ})_{3}\right.$ bipy $]$ - комплекс, содержащий положительно заряженный парамагнитный $\mathrm{Eu}^{3+}$ с парамагнитными лигандами SQ (SQ - анион-радикал 3,6-ди-трет-бутилбензохинона) и диамагнитным bipу (бипиридил).

Высокоспиновые элементоорганические магнетики можно использовать для создания электрических проводников, магнитных переключателей, для элементов памяти квантовых компьютеров, для спинтронных устройств [13-18]. Комплексы трехвалентного европия проявляют эффект усиления магнитного дипольного излучения для видимого и ультрафиолетового диапазонов [19]. Использование их в качестве усилителя в радиочастотном диапазоне расширило бы спектр их практического применения. Именно поэтому отработка проведения процесса СИ в новом режиме, а именно в режиме „СИ при реологическом взрыве разгрузки“ (режим RSI при RVR), т. е. при резком сбросе давления, действующего на образец композита, и выяснения, какие физико-химические процессы протекают в полимерной матрице при этом - актуальная задача. Следует отметить, что проведение экспериментов в данном режиме обеспечивает более широкие экспериментальные возможности для исследования механической активации различных твердофазных объектов, в том числе по- 

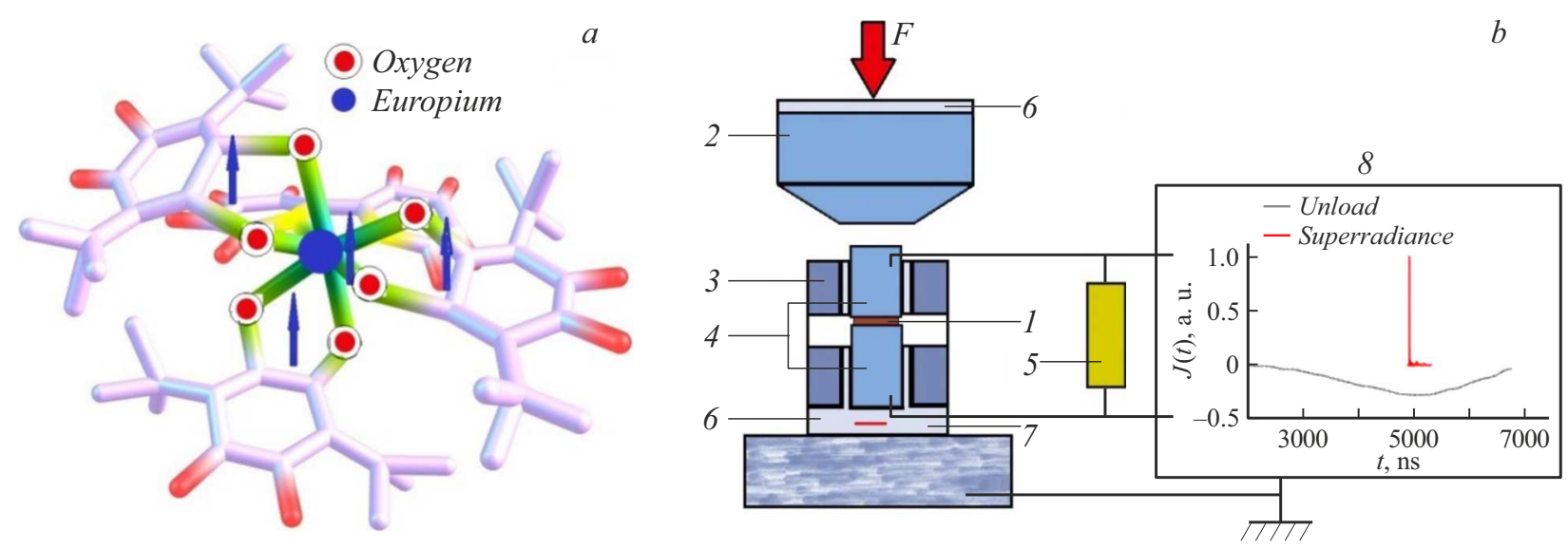

Рис. 1. $a-$ структура комплекса $\mathrm{Eu}(\mathrm{III})(\mathrm{SQ}){ }_{3}$ bipy; $b-$ схема ячейки высокого давления. 1 - образец; $2-$ прессовое оборудование; 3 - стальная обойма; 4 - пуансоны; 5 - сопротивление нагрузки; 6 - изоляция; 7 - пьезодатчик; 8 - цифровой осциллограф.

лимерных композитов. Эти исследования закладывают основу создания нового метода синтеза и исследования композитов, реализующих трансформацию механической энергии в СИ в радиочастотном диапазоне и формирование при этом импульса свободных электронов, которые инициируют окислительно-восстановительные процессы, протекающие в полимерной матрице.

Для решения этих задач в настоящей работе использовали комплекс $\left[\mathrm{Eu}^{\mathrm{III}}(\mathrm{SQ})_{3}\right.$ bipy] (структура приведена на рис. $1, a)$. Методика синтеза и структура данного комплекса приведены в работе [12]. Так же в работе [12] показано, что данный комплекс - это молекулярный магнетик, имеющий четыре парамагнитных центра ион $\mathrm{Eu}(\mathrm{III})$ и три - SQ лиганда (рис. $1, a)$.

\section{1. Экспериментальная часть}

Синтезированы образцы композитов С1, C2, C3, C4, $\mathrm{C} 5$, содержащие $0.4 \cdot 10^{20}, 0.6 \cdot 10^{20}, 0.8 \cdot 10^{20}, 1.0 \cdot 10^{20}$, $1.2 \cdot 10^{20}$ комплексов $\mathrm{Eu}(\mathrm{III})(\mathrm{SQ})_{3}$ bipу в $1 \mathrm{~cm}^{3}$ полистирола (PS) соответственно. Смешивание $\left[\mathrm{Eu}^{\mathrm{III}}(\mathrm{SQ})_{3}\right.$ bipy] и PS осуществляли в шаровой вибрационной микромельнице с агатовой гарнитурой Pulverisette 0 (Германия) в течение $10 \mathrm{~min}$. Полученную смесь прессовали при $190^{\circ} \mathrm{C}$. Образцы диаметром $14 \mathrm{~mm}$ и толщиной $1 \mathrm{~mm}$ не испытывали реологического взрыва при одноосном сдавливании до $5 \mathrm{GPa}$. Для исследования возникновения импульсов СИ при механическом воздействии использовали специальную ячейку высокого давления, которая помещалась в машину сжатия ИС-500. Схема установки приведена на рис. $1, b$.

Были выбраны следующие режимы RSI при RVR на образец вначале действует одноосная нагрузка (скорость сдавливания $1.0 \mathrm{GPa} / \mathrm{s})$, а затем следует резкий сброс давления при заданном $P_{\mathrm{RVR}}=2$ или $3 \mathrm{GPa}$. Сброс давления производился автоматически, для этого в конструкцию машины сжатия ИС-500 было внесено авторское изменение. Видно, что ячейка, изолированная от прессового оборудования, состоит из наковальни Бриджмена, стальной обоймы и пуансонов, между которыми находится исследуемый образец. В изоляцию вмонтирован планарный пьезодатчик для измерения давления на образец. Пуансоны через сопротивление нагрузки $R=50 \Omega$ соединены с двухканальным цифровым осциллографом Tektronix MSO 200, к которому также подключен и пьезодатчик. Ячейка позволяет регистрировать импульс тока $J(t)$, генерируемый электрической составляющей $E(t)$ электромагнитного излучения, возникающего в образце. Соответственно на осциллографе регистрируется импульс напряжения $U(t)=R J(t)$ (на рис. $1, b$ это узкий сигнал). Также при необходимости пьезодатчиком фиксируется и процесс сброса давления, которому отвечает более протяженный импульс разгрузки, сигнал которого приведен на рис. $1, b$ черным цветом.

Для получения спектров ЭПР использовали ЭПР спектрометр Bruker EMX (Германия). Электронные микрофотографии и электронограммы получены с помощью туннельного электронного микроскопа LEO 912 AB OMEGA („Zeiss“‘ Германия).

\section{2. Теоретическая часть}

СИ - это процесс электромагнитного излучения среды как единого сфазированного ансамбля (макродиполя), состоящего из микродиполей, охваченных коллективным взаимодействием через кооперативную волну связи. Интенсивность импульса СИ $W_{S} \propto N^{2}$, где $N-$ число центров возбуждения. Для лазеров интенсивность излучения пропорциональна $N$, т. е. $W_{L} \propto N$. Коллективная динамика сверхизлучательного состояния характеризуется коэффициентом связи $\left(\omega_{C}\right)^{2}$, который является показателем интенсивности обменно-волнового процесca, обеспечивающего коллективную сфазированность колебаний всех микродиполей сверхвозбужденной среды. 
Кооперативная волна с частотой $\omega_{C}$, промодулированная электрической волной, формирует стоячую волну в заданном объеме среды вблизи собственной резонансной частоты $\omega_{0}$ возбужденной среды, соответствующей одному из уровней возбужденной системы. При этом имеет место поляритонный резонанс - при поляризации на резонансной частоте возникающие в среде волны поляризации охватывают все микродиполи, образуя единый сфазированный макродиполь. Энергия поля поляризации и фазы волн поляризации становятся едиными для всех центров возбуждения (возбужденных атомов и молекул). Сверхизлучательное коллективизированное состояние характеризуется общими энергетическими уровнями. Описание излучения вследствие переходов между этими уровнями в макроскопическом приближении возможно классическими уравнениями Максвелла (1) и (2) и уравнением для поляризации $P(3)$, а также уравнением (4), полученным Дике [6]:

$$
\begin{gathered}
\operatorname{rot} E=-\frac{1}{c} \frac{\partial B}{\partial t}, \\
\operatorname{rot} B=-\frac{1}{c} \frac{\partial(E+4 \pi P)}{\partial t}+\frac{4 \pi \sigma}{c} E, \\
\frac{\partial^{2} P}{\partial t^{2}}+\omega_{r, E} \frac{\partial P}{\partial t}+\omega_{r e z, E} P=\omega_{c}^{2} E, \\
\frac{d\left(R_{8}\right)}{d t}=-\frac{1}{T_{1}}\left(\frac{N^{2}}{2}-\frac{\left(R_{8}\right)^{2}}{2}+N+\left\langle R_{3}\right\rangle\right),
\end{gathered}
$$

где $T_{1}-$ время энергетической релаксации, $\omega_{r, E}=1 / T_{2}$, $T_{2}$ - время фазовой релаксации активных центров, $\left\langle R_{3}\right\rangle$ - среднее значение разности населенности уровней в единичном объеме среды, содержащем $N$ возбуждаемых атомов и $n$ возбужденных атомов. Таким образом, $\left\langle R_{3}\right\rangle=n-(N-n)=2 n-N$. Если правую часть уравнения (4) переписать в виде $1 / 2\left[(N+1)^{2}-n-\left(\left\langle R_{3}\right\rangle-1\right)^{2}\right]$, то

$$
\left\langle R_{3}\right\rangle=1-(N+1) \text { th }\left[\frac{N+1}{2 T_{1}}\left(t-t_{0}\right)\right] .
$$

При $\left\langle R_{3}(t=0)\right\rangle=N$ имеем $t_{0}=\frac{T_{1}}{N+1} \ln N$ и получаем мощность электромагнитного излучения (интенсивность излучения) как функцию времени

$$
I(t)=I_{0} N^{2} \operatorname{sech}^{2}\left[\frac{1}{2 \tau_{c}}\left(t-t_{0}\right)\right],
$$

$I(t)$ описывает импульс СИ, мощность которого $I_{\max }\left(t=t_{0}\right)$ достигает максимума при $t=t_{0}$, а $I_{0}-$ интенсивность излучения одной частицы из ансамбля $N$ частиц. Величина максимума пропорциональна $N_{2}$, $t_{0}-$ время задержки импульса $t_{0}=2 \tau_{c} \ln N, \tau_{c}-$ ширина импульса на полувысоте $\left(2 \tau_{c}-\right.$ длительность импульса).

Более подробно уравнения (1)-(6) обсуждаются в работах $[6,19-21]$. Заметим, что уравнение (3) описывает систему в рамках модели Лоренца для гармонического осциллятора с затуханием [22], когда вынужденные колебания заряженных частиц приводят к поляризации среды, которая для не взаимодействующих частиц одного типа описывается вектором поляризации $\mathbf{P}$, равным $N \mathbf{p}$, где $N-$ концентрация частиц, а $\mathbf{p}=\mathbf{q r}-$ дипольный момент одной частицы. При этом если изменение электрического дипольного момента и изменение в структуре спиновой системы связаны между собой, т.е. электрический дипольный момент $D$ связан со спиновой системой и $D \propto R \times\left(S_{a} \times S_{b}\right)$ [23], где $\mathbf{R}-$ относительное положение двух произвольно взятых спинов в исследуемом комплексе, то, поскольку $\mathbf{R}$ изменяется во времени, соответственно изменяются векторы дипольных моментов D и векторы поляризации $\mathbf{P}$ и, как следствие, магнитные моменты системы М, которые связаны со спиновым ансамблем. Тогда подобная система описывается как в рамках модели Лоренца для гармонического осциллятора с затуханием [22] - уравнение (3), так и в рамках модели на основе уравнения Ландау-БлохаБломбергена [24-26], т. е. уравнения (7):

$$
\frac{d M}{d t}=-\gamma M \times H+\omega_{r, H}\left[\chi_{0} H-M\right] .
$$

Соответственно анализировать спектры, полученные после фурье-преобразования сигналов электрических импульсов $E(t)$ для подобных систем можно, используя приведенное для $\varepsilon^{\prime \prime}(\omega)$ уравнение (3) и приведенное для $\chi^{\prime \prime}(\omega)$ уравнение $(7)$, т. е. по формулам $(8)[27,28]$

$$
\begin{gathered}
\varepsilon^{\prime \prime}(\omega)=\varepsilon_{0} \frac{2 \omega_{r, E} \omega_{C}^{2} \omega}{\left(\omega_{r e z, B}^{2}-\omega^{2}\right)^{2}+4 \omega_{r, E}^{2} \omega^{2}}, \\
\chi^{\prime \prime}(\omega)=\chi_{0} \frac{2 \omega_{r, H} \omega_{H} \omega^{2}}{\left(\omega_{r e z, H}^{2}-\omega^{2}\right)^{2}+4 \omega_{r, H}^{2} \omega^{2}},
\end{gathered}
$$

где $\omega_{r, E}=\Delta E(\mathrm{MHz}) / 2 \quad$ и $\quad \omega_{r, H}=\Delta H(\mathrm{MHz}) / 2$, а $\Delta E(\mathrm{MHz})$ и $\Delta H(\mathrm{MHz}) \quad-$ полуширины резонансных линий. При этом $\omega_{r e z, E}^{2}=\omega_{r, E}^{2}+\omega_{C}^{2}$ и $\omega_{r e z, H}^{2}=\omega_{r, H}^{2}+\omega_{H}^{2}, \omega_{H}=\gamma H$.

\section{3. Результаты и обсуждение}

В проводимых в настоящей работе экспериментах с помощью осциллографа регистрировали переменное напряжение $U(t)=R J(t)$. Ток $J(t)$ генерируется электрической составляющей $E(t)$ электромагнитного излучения, которое возникает в исследуемых образцах при механическом воздействии и $U(t) \propto J(t) \propto E(t)$. При реологических взрывах при 3 и $2 \mathrm{GPa}$ были зафиксированы интенсивные сигналы от образцов композитов $\mathrm{C} 1-\mathrm{C} 5$ и зафиксированы слабые сигналы от матричного полимера PS. В отнормированном к максимальному по интенсивности сигналу виде сигналы от $\mathrm{C} 1-\mathrm{C} 5$ и от матричного полимера представлены на рис. $2, a$. Самый интенсивный сигнал в $500 \mathrm{~V}$ наблюдался для С5 при $3 \mathrm{GPa}$. Интенсивность сигнала от PS, записанного при 

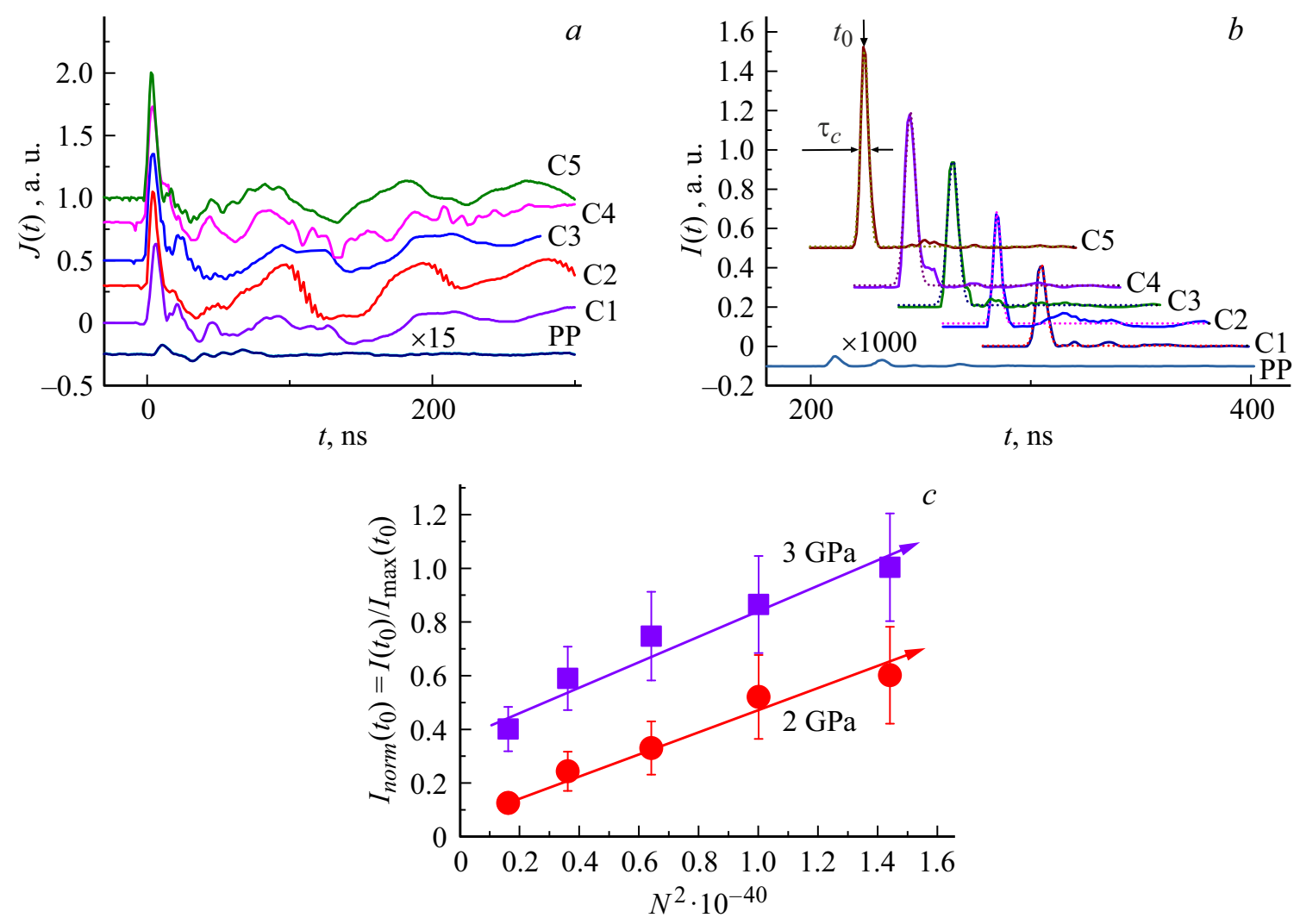

Рис. 2. $a-$ временная развертка нормированных сигналов $J(t)$ для композитов $\mathrm{C} 1-\mathrm{C} 5$ и $\mathrm{PS} ; b-$ временная развертка нормированных сигналов $I(t) \propto[E(t)]^{2}$ для композитов C1-C5 и PS; $c$ - нормированная зависимость $I\left(t_{0}\right)$ от квадрата концентрации комплексов $\mathrm{Eu}(\mathrm{III})(\mathrm{SQ})_{3}$ bipy в $1 \mathrm{~cm}^{3}$ для композитов $\mathrm{C} 1-\mathrm{C} 5$. Нормировка проведена к максимальному значению $I\left(t_{0}\right)$ для С5.

тех же условиях, не превышала $15 \mathrm{~V}$. Из представленного рисунка видно, что сигналы от композитов имеют характерный интенсивный узкий пик в самом начале. Подобный пик отсутствует в сигналах от полистирола.

На рис. 2, $b$ приведены импульсы электромагнитного излучения $I(t) \propto[E(t)]^{2} \propto[J(t)]^{2} \propto[U(t)]^{2}$, достигающие максимальной интенсивности при $t=t_{0}$. Видно, что форма линии полос излучения соответствует закону, характерному для процессов СИ - экспоненциальному симметричному подъему и спаду (на рис. $2, b$ анаморфозы по формуле (6) даны пунктиром ). Установлено, что интенсивность пиков $I\left(t_{0}\right)$ (при $t=t_{0}$ ) пропорциональна квадрату концентрации комплексов $N^{2}$. Это хорошо видно из рис. 2, $c$, на котором показана зависимость нормированной амплитуды $I_{n o r m}\left(t_{0}\right)=I\left(t_{0}\right) / I_{\max }\left(t_{0}\right)$ от $N^{2}$ для комплексов $\left[\mathrm{Eu}^{\mathrm{III}}(\mathrm{SQ})_{3}\right.$ bipy]. $I_{\max }\left(t_{0}\right)$ соответствует максимальному значению импульса RSI для композита С5 при $3 \mathrm{GPa}$, где $I_{\max }\left(t_{0}\right)$ для образца, содержащего $1.2 \cdot 10^{20}$ комплексов $\left[\mathrm{Eu}^{\mathrm{III}}(\mathrm{SQ})_{3}\right.$ bipy] в $1 \mathrm{~cm}^{3} \mathrm{PS}-$ т.е. с максимальной концентрацией комплекса европия. Как следует из рис. 2, $c$, использование режима RSI при RVR позволило установить, что процесс RSI можно реализовывать для всех образцов С1-C5 при $P_{\mathrm{RVR}}$ как при $2 \mathrm{GPa}$, так и при $3 \mathrm{GPa}$. Следует подчеркнуть, что интенсивность пиков $I\left(t_{0}\right)$ от образцов композитов
C1-C5 почти на четыре порядка превосходит интенсивность импульса от образца „чистого“ $\mathrm{PS}$ - матричного полимера при реализации в нем реологического взрыва разгрузки при $3 \mathrm{GPa}$; также эти характерные пики со структурой, описываемой формулой (6), отсутствуют в сигналах матричного полимера (рис. 2, $b$ ). Для определения $t_{0}=2 \tau_{c} \ln N$ из эксперимента брали $\tau_{c}$ (считая, что $\tau_{c}$ - ширина импульса на полувысоте, а $2 \tau_{c}-$ длительность импульса); отметим, что количество парамагнитных комплексов в образцах, превратившихся в непарамагнитные частицы - это 70\% от комплексов во всех образцах и линейно пропорционально $N$ - количеству комплексов, введенных в композит при синтезе в пересчете на $\mathrm{cm}^{-3}$. Установлено, что время задержки импульса уменьшается с увеличением концентрации комплексов в образце, и в нашем случае для С1-C5 оно равно $300,280,260,240,200 \mathrm{~ns}$ соответственно (рис. $2, b)$.

Фурье-анализ импульсов $J(t) \propto E(t)$ показал, что наблюдаемые процессы испускания RSI лежат в диапазоне частот от 0 до $300 \mathrm{MHz}$ - т.е. в радиочастотном диапазоне (рис. $3, a$ ). Как видно из рис. $3, a$, фурье-образы сигналов $E(t) \propto J(t)$ имеют вид полосатых (полосчатых) спектров. При этом нормированный фурье-спектр для композита (на рис. 3, $a$ спектр 1) кардинально отли- 

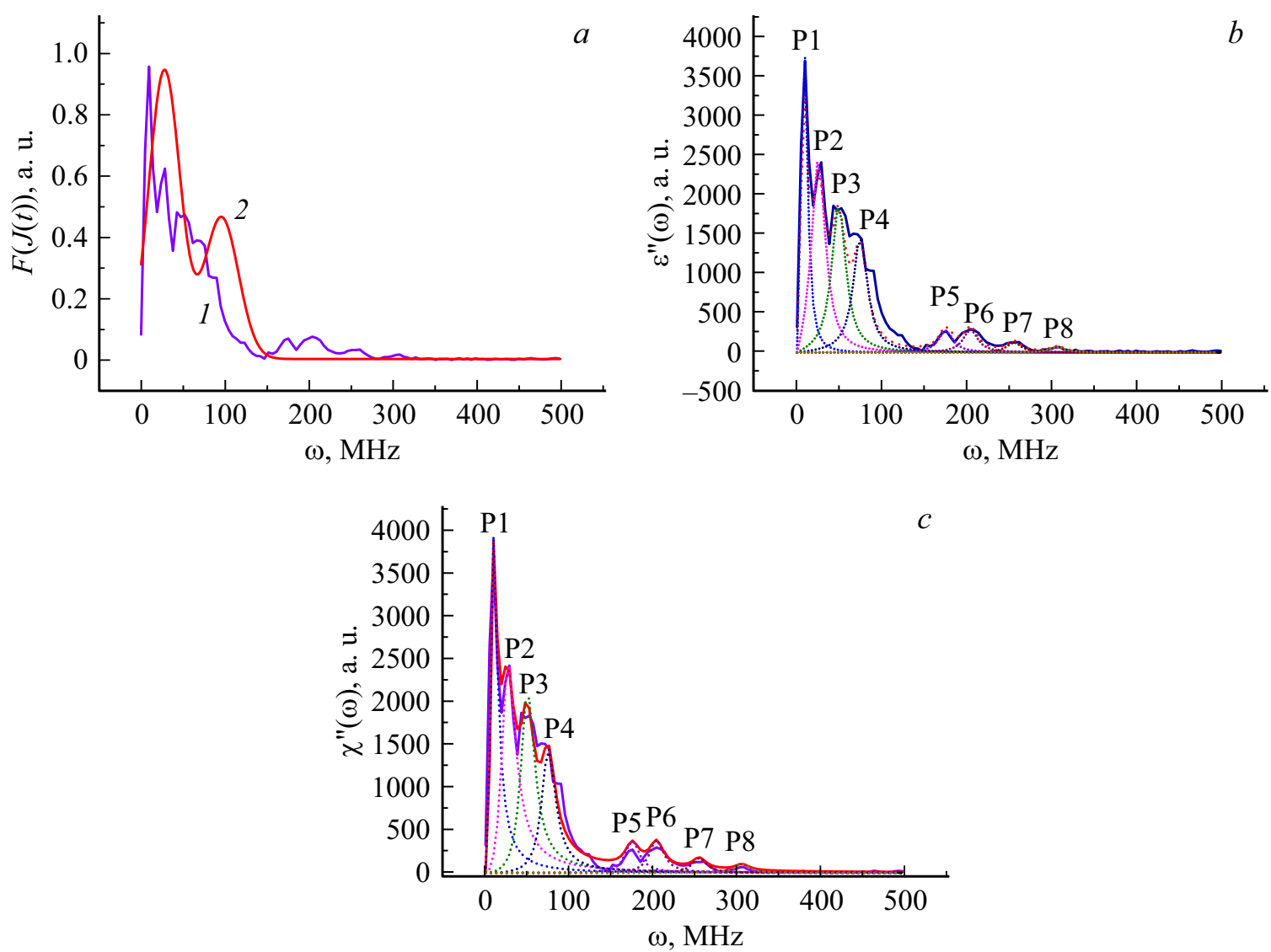

Pис. 3. $a-$ нормированный фурье-образ электрического сигнала для композита C5 (1) и PS (2); $b-$ фурье-образ электрического сигнала для композита С5 и полосы излучения, полученные по методу Лоренца (их параметры даны в табл. 1); $c$ - фурье-образ электрического сигнала для композита С5 и полосы излучения, полученные по методу Ландау-Блоха-Бломбергена (их параметры даны в табл. 1).

чается по структуре от нормированного фурье-спектра матричного полимера - PS (на рис. 3, спектр 2). T.е. можно утверждать, что фурье-спектр обусловлен введенным комплексом $\left[\mathrm{Eu}^{\mathrm{III}}(\mathrm{SQ})_{3}\right.$ bipy]. Как известно, полосатые (полосчатые) спектры излучаются отдельными возбужденными молекулами, не связанными или слабо связанными друг с другом. Излучение вызвано как электронными переходами в атомах, так и колебательными движениями самих атомов в молекулах. Поэтому можно представить, что при воздействии импульса упругих волн (ИУВ) происходят локальные размораживания подвижности фрагментов макромолекул и дестабилизация введенных в полимерную матрицу парамагнитных комплексов, а также реализуются процессы ионизации и образование ансамбля свободных электронов. При этом возникает электромагнитное излучение на частотах, обусловленных собственными колебаниями зарядов. Поскольку высокочастотные колебания $E(t)$ связаны с колебаниями зарядов, свободных и связанных с молекулярными компонентами композита, они, очевидно, обусловлены колебаниями поляризации. Поскольку подобные многоспиновые объекты изменяют свои магнитные характеристики при воздействии давления, очевидно, что у них электрический дипольный момент $D$ связан с их спиновой системой, т.е. $D \propto R \times\left(S_{a} \times S_{b}\right)$, где $R$ - относительное положение двух произвольно взятых спинов в комплексе $\left[\mathrm{Eu}^{\mathrm{III}}(\mathrm{SQ})_{3}\right.$ bipy]. Поскольку $R$ изменяется во времени, соответственно изменяются векторы дипольных моментов $D$ и векторы поляризации $P$ и соответственно магнитные моменты системы $M$, которые связаны со спиновым ансамблем [23]. Результаты расчетов спектра Фурье для С5 при $3 \mathrm{GPa}$ по формулам (9) для отдельных полос излучения, из которых формируется общий спектр, приведены на рис. 3, b, c. Исходные данные для расчетов даны в табл. 1.

Однако анализ сигналов от реологического взрыва композитов $\mathrm{C} 1-\mathrm{C} 5$ и матричного полимера на основе уравнений (8) не позволяет оценить релаксационные свойства изучаемых систем. Провести анализ этих характеристик позволяет метод Гаврилиака-Негами [29]. Поскольку формализм описания спектров в рамках метода Гаврилиака-Негами работает с одиночной линией спектра независимо от того, принадлежит эта линия процессу излучения или поглощения, полосы радиочастотного СИ можно анализировать с помощью метода 
Таблица 1. Параметры полос излучения

\begin{tabular}{l|c|c|r|r|r|r|r|r}
\hline$\omega_{\text {rez } . E}(\mathrm{MHz})$ & 9.50 & 27.00 & 50.00 & 75.00 & 175.00 & 204.00 & 255.00 & 306.00 \\
\hline$\omega_{r . . E}(\mathrm{MHz})$ & 5.00 & 10.00 & 10.00 & 10.00 & 10.00 & 10.00 & 10.00 & 10.00 \\
\hline$\omega_{C}(\mathrm{MHz})$ & 8.07 & 25.07 & 48.98 & 71.33 & 174.71 & 203.75 & 254.80 & 305.83 \\
\hline$\varepsilon_{0}$ & 4061.53 & 1588.5 & 596.92 & 320.00 & 31.70 & 29.08 & 10.03 & 4.70 \\
\hline$\omega_{\text {rez } . H}(\mathrm{MHz})$ & 9.50 & 27.00 & 50.00 & 75.00 & 175.00 & 204.00 & 255.00 & 306.00 \\
\hline$\omega_{r . H}(\mathrm{MHz})$ & 5.00 & 10.00 & 10.00 & 10.00 & 10.00 & 10.00 & 10.00 & 10.00 \\
\hline$\omega_{H}(\mathrm{MHz})$ & 8.07 & 25.07 & 48.98 & 71.33 & 174.71 & 203.75 & 254.80 & 305.83 \\
\hline$\chi_{0}$ & 4625 & 1389 & 548.95 & 279.3 & 31.80 & 35.00 & 12.91 & 4.62
\end{tabular}

Таблица 2. Параметры полос фурье-спектра для полос СИ для С5 и для полос фурье-спектра PS (модель Гаврилиака-Негами)

\begin{tabular}{c|c|c|c|c|c}
\hline Полоса спектра СИ & $\log \omega_{0}$ & $\Delta \varepsilon$ & $a$ & $b$ & $\tau_{0}, \mathrm{~ns}$ \\
\hline P1 & 7.616 & 1.289 & 1.235 & 1.320 & 28 \\
P2 & 8.202 & 0.366 & 1.631 & 1.025 & 5.2 \\
P3 & 8.488 & 0.172 & 1.777 & 1.005 & 3.0 \\
P4 & 8.668 & 0.096 & 1.844 & 1.002 & 2.3 \\
P5 & 9.039 & 0.0077 & 1.930 & 1.000 & 0.9 \\
P6 & 9.106 & 0.0073 & 1.940 & 1.000 & 0.8 \\
P7 & 9.203 & 0.0025 & 1.951 & 1.000 & 0.6 \\
P8 & 9.286 & 0.0011 & 1.959 & 1.000 & 0.5 \\
PS_1 & 8.295 & 12.331 & 0.919 & 8.471 & 12.0 \\
PS_2 & 8.032 & 2.229 & 1.576 & 1.579 & 27.0
\end{tabular}

Гаврилиака-Негами по формулам (9) и (10) [29]:

$$
\begin{gathered}
\varepsilon^{\prime \prime}=\frac{\Delta \varepsilon}{\left[1+\left(i \omega \tau_{0}\right)^{a}\right]^{b}}, \\
g(\tau)=\frac{1}{\pi} \frac{\left(\tau / \tau_{0}\right)^{a b} \sin (b \theta)}{\left[\left(\tau / \tau_{0}\right)^{2 a}+2\left(\tau / \tau_{0}\right)^{a} \cos (a \pi)+1\right]^{b / 2}},
\end{gathered}
$$

где $\theta=\arctan \left|\frac{\sin (b \pi)}{\left(\tau / \tau_{0}\right)^{a}+\cos (a \pi)}\right|$, и получить коэффициенты $\log \omega_{0}$ ( $\omega_{0}-$ частота в максимуме полосы излучения), $\Delta \varepsilon\left(\Delta \varepsilon=\varepsilon_{s}-\varepsilon_{\infty}\right.$, где $\varepsilon_{s}$ и $\varepsilon_{\infty}-$ проницаемость при $\omega=0$ и $\omega \gg 1 / \tau_{0}$ соответственно), $a$ (определяет степень частотной зависимости), $b$ (определяет ширину спектра), позволяющие аппроксимировать полосы излучения (рис. $4, a, b)$, а также определить характерные значения времени релаксации в максимумах полос излучения $\tau_{0}=1 /\left(2 \pi \tau_{0}\right)$. Аппроксимация фурье-образа электрического сигнала для композита С5 и для полистирольной матрицы по модели Гаврилиака-Негами показана на рис. $4, a, b$, а данные, полученные при этом, приведены в табл. 2.

Расчет кривых релаксации проведен по формулам (6) и данным из табл. 2 ; значения $\tau_{0}$ совпадают с максимумами кривых релаксации на рис. $4, c$.
Используя значения коэффициентов $\log \omega_{0}, \Delta \varepsilon, a$, $b$, из табл. 2 можно построить кривые распределения времен релаксации $g(\tau)$ для полос композита (С5 в нашем случае) и для полистирольной матрицы (эти кривые приведены на рис. 4,c), а также определить характерные значения времен релаксации в максимумах полос излучения $\tau_{0}$ (приведены в табл. 2). Видно, что распределения времен релаксации имеют длинный хвост со стороны больших частот. Также из рис. 4, $c$ видно, что релаксационные кривые для PS (кривая PS_1) и для композита (кривая Р1) совпадают. Это указывает на непосредственное участие полимерной матрицы в исследуемых процессах и свидетельствует о том, что колебания полистирольной матрицы активируют поляризационные колебания комплекса европия.

Очевидно, что быстрые процессы связаны и обусловлены нестабильными короткоживущими продуктами, имеющими времена жизни порядка сотен наносекунд. Представляет интерес выяснить, к каким стабильным продуктам приводят быстропротекающие физико-химические процессы с участием короткоживущих частиц.

Методами ЭПР и ТЭМ были проведены оценки количества стабильных частиц после реализации процесса СИ и определены вероятные стабильные продукты этих превращений.

Анализируя спектры ЭПР образцов композитов $\mathrm{C} 1-\mathrm{C} 5$ (рис. $5, a, b)$, можно уверенно утверждать, что они представляют собой суперпозицию двух спектров ЭПР. Один из этих спектров принадлежит иону европия (с $g_{1}=2.13, g_{2}=1.98, g_{3}=1.87$ и соответственно с $\left.g_{\text {med }}=1.99\right)$, а другой, отмеченный буквой $\mathrm{A}$, скорее всего, принадлежит бирадикалу (или трирадикалу) на лигандах комплекса $\left[\mathrm{Eu}^{\mathrm{III}}(\mathrm{SQ})_{3}\right.$ bipy $]$ - более определенно соотнести этот парамагнитный центр (ПМЦ) не представляется возможным, так как низкополевые сигналы ЭПР, характерные для бирадикалов (или трирадикалов) [30], выявить не удалось. После протекания механохимических процессов (которые сопровождаются актом испускания СИ) фиксируется суммарный спектр ЭПР, который представлен суперпозицией синглетного спектра с $g_{\mathrm{med}}=1.98$ и 

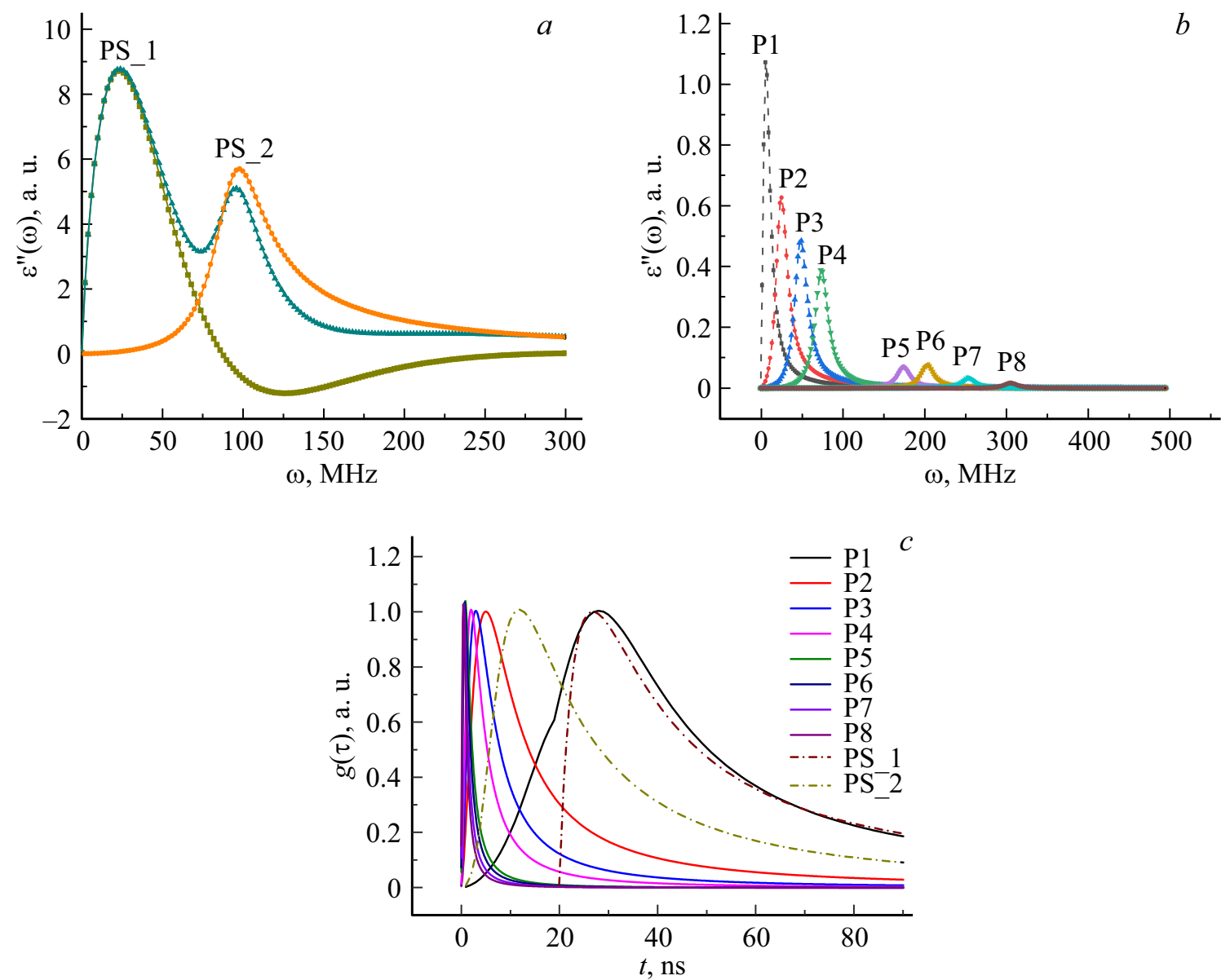

Pис. 4. $a$ - фурье-образ электрического сигнала для PS и полосы излучения, полученные по методу Гаврилиака-Негами (параметры даны в табл. 2); $b$ - аппроксимация фурье-образа электрического сигнала для композита С5 по модели Гаврилиака-Негами (параметры полос даны в табл. 2); $c$ - кривые распределения времен релаксации $g(\tau)$ от $t$ для PS-матрицы и композита С5. Номера полос излучения и кривых $g(\tau)$ от $\tau$ соответствуют номерам в табл. 2.

спектра от бирадикала (или трирадикала) (рис. 5,c). Подобную трансформацию спектров ЭПР можно, вероятно, трактовать следующим образом: происходит удаление - механохимический отрыв бипиридинового лиганда $\left[\mathrm{Eu}^{\mathrm{III}}(\mathrm{SQ})_{3}\right.$ bipy $] \rightarrow\left[\mathrm{Eu}^{\mathrm{III}}(\mathrm{SQ})_{3}\right]+$ bipy и ион европия стабилизируется в кислородном октаэдрическом окружении, при этом сохраняются неспаренные электроны на SQ-лигандах. Подобный процесс - это одна из стадий механохимических превращений исходного $\left[\mathrm{Eu}^{\mathrm{III}}(\mathrm{SQ})_{3}\right.$ bipy]. В пользу того, что до и после механической активации мы методом ЭПР фиксируем только парамагнитные продукты, говорит то, что спектры ЭПР, записанные при комнатной температуре, уширились (рис. $5, b, d$ ), т. е. они связаны с парамагнитными комплексами $\mathrm{Eu}(\mathrm{III})$. В случае, если бы в образцах образовались суперпарамагнитные частицы, вероятно, соответствующие им линии ЭПР должны были бы испытать сужение, как фиксировали в работах для наночастиц $\mathrm{Co}, \mathrm{Fe}, \mathrm{Ni}$ [31]. Именно поэтому было проведено двойное интегрирование спектров, записанных при $77 \mathrm{~K}$ (интегрирование проводили по всем ПМЦ в образце - от ПМЦ на $\mathrm{Eu}(\mathrm{III})$ и на лигандах), позволившее установить, что после реологического взрыва во всех образцах (в пересчете на количество ПМЦ в $\mathrm{cm}^{3}$ ) остается только 30\% ПМЦ от исходного количества комплексов $\left[\mathrm{Eu}^{\mathrm{III}}(\mathrm{SQ})_{3}\right.$ bipy]. Более детальный анализ методом ЭПР комплексов [Eu ${ }^{\mathrm{III}}(\mathrm{SQ})_{3}$ bipy] и их механохимических превращений требует более высоких экспериментальных возможностей и отдельной публикации.

Также были проведены исследования с помощью просвечивающего электронного микроскопа LEO 912 AB OMEGA при ускоряющем напряжении $100 \mathrm{kV}$ и установлено образование металлических наночастиц европия.

На рис. 6 приведены микрофотографии $(a, b, d, e)$ и электронограммы $(c, f)$ образцов композитов $\left(\mathrm{PS}+\mathrm{Eu}(\mathrm{III})(\mathrm{SQ})_{3}\right.$ bipy) $\mathrm{C} 1$ и $\mathrm{C} 5$, до $(a, d)$ и после $(b, e)$ механической активации при $3 \mathrm{GPa}$. Полученные изображения и электронограммы демонстрируют возникновение металлических наночастиц европия (рис. $6, b, c, e, f)$. Размытое кольцо для композита С1 (рис. $6, c$ ) соответствует дифракции от очень мелких наночастиц размером менее $1 \mathrm{~nm}$. Для композитов при 

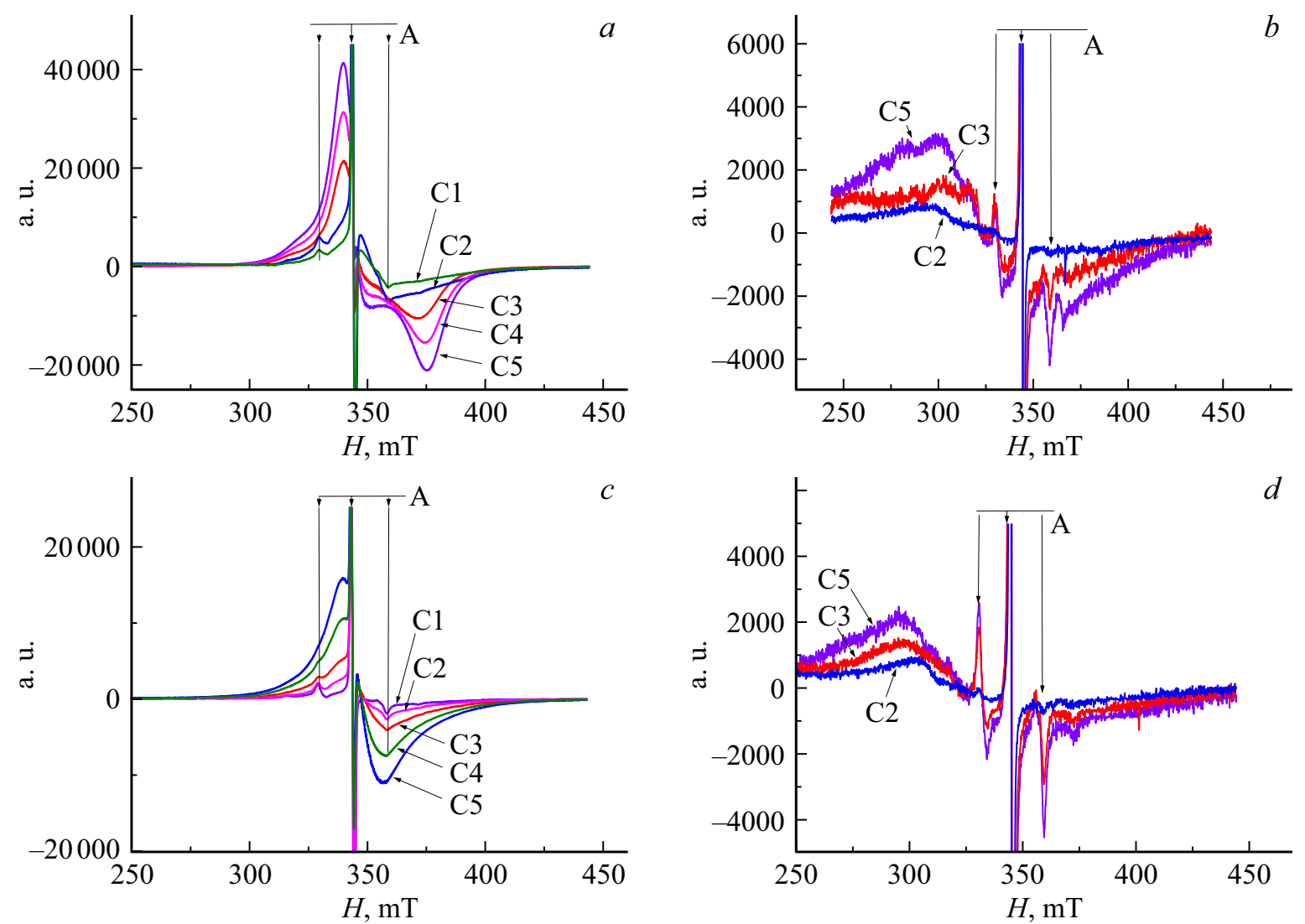

Рис. 5. Спектры ЭПР, записанные при $77 \mathrm{~K}(a, c)$ и при комнатной температуре $(b, d)$ для композитов до $(a, b)$ и после $(c, d)$ реологического взрыва.
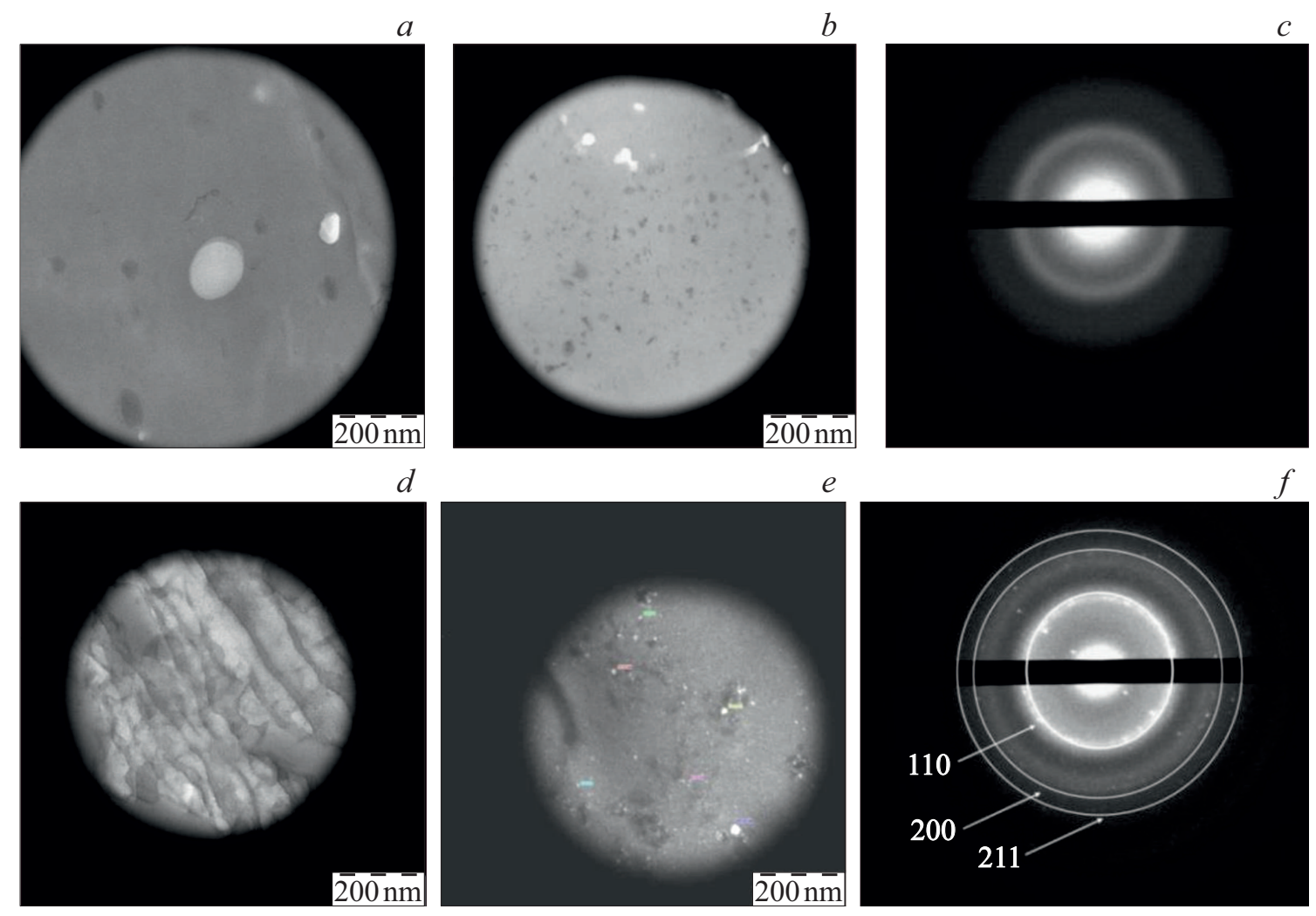

Рис. 6. Микрофотографии $(a, b, d, e)$ и электронограммы $(c, f)$ образцов композитов $\mathrm{C} 1(a-c)$ и $\mathrm{C} 5(d-f)$. 
увеличении концентрации исходного [Eu ${ }^{\mathrm{III}}(\mathrm{SQ})_{3}$ bipy] наблюдается наличие металлических монокристаллов Еu размера $5 \mathrm{~nm}$ и более, о чем можно судить по наличию отдельных дифракционных рефлексов на электронограмме (рис. 6,f). Дифракционная картина электронов совпадает с таковой для металлического европия, что подтверждает наличие рефлексов [110], [200], [211] (рис. 6, $f$ - белые круги) и доказывает наличие нанокристаллов металлического Еu в образце.

\section{Заключение}

Можно полагать, что процесс механической активации композитов протекает при непосредственном участии полимерной матрицы, поскольку колебания цепей полимерной матрицы приводят к активации металлоорганических комплексов. При этом разумно предположить, что активация (механоактивация) наиболее активно происходит в нанообъемах (нанополостях) с подвижными стенками, возникающих при реологическом взрыве в полимерной матрице [10]. Очевидно, что эти нанополости возникают в местах соприкосновения (нахождения) наполнителя и полимерных цепей PS. В активированных таким образом комплексах начинают протекать физикохимические процессы, в том числе окислительно-восстановительные, связанные с редокс-активными лигандами, переносом электронной плотности, образованием подвижных электронов и возникновением триплетных возбужденных состояний и отрицательной спиновой поляризации [32-35]. При этом четырехспиновая система трансформируется в трехспиновую с образованием подвижного электрона $\mathrm{e}^{-}$, который инициирует процессы восстановления ионов европия и последующие процессы синтеза наночастиц металлического европия. Образование неспаренных свободных электронов в образце можно рассматривать как исходный этап сложного, вероятно, каталитического процесса, приводящего к образованию металлических наночастиц европия в полимерной матрице. Очевидно, что подобные утверждения требуют проведения дополнительного комплексного исследования этих физико-химических процессов с учетом специфики механохимических процессов при наличии полимерной матрицы. Действительно, аномальную диффузионную подвижность реагентов при механической активации отмечали достаточно давно [36]. В более поздней работе [10] доказано возникновение свободных нанополостей в полистирольной матрице при реологическом взрыве. Опираясь на эти работы и данные о количестве свободных электронов, возникающих при механоактивации, и количестве комплексов $\mathrm{Eu}(\mathrm{III})$ в исходных образцах, можно предположить, что механохимические превращения в полимерной матрице идут в нанореакторах при аномально высокой диффузионной подвижности компонентов. Именно это позволяет наблюдать приведенные в работе эффекты. Представленные в работе эффекты и ранее опубликованная авторами работа [10] являются логическим продолжением работ С.Н. Журавлева и В.Р. Регеля [37,38], в которых рассмотрены процессы образования ансамблей нано- и микротрещин, несущих электрические заряды. Очевидно, что рассмотрение описанных в работе физико- химических процессов требует отдельной публикации и проведения дополнительных экспериментов с целью развития и обобщения воззрений, изложенных в работах [33-39].

Уже сейчас можно отметить, что важной особенностью подобного механохимического подхода является возможность эффективного контроля за размером синтезируемых наночастиц в полимерной матрице в зависимости от состава композита. Также отметим, что системы на основе подобных элементоорганических соединений являются хорошей моделью для исследования механохимических процессов синтеза полимерных композитов.

\section{Благодарности}

Авторы выражают благодарность Центру коллективного пользования ИБХФ РАН за помощь в получении данных ЭПР.

\section{Финансирование работы}

Работа выполнена при финансовой поддержке Минобрнауки России (грант № 075-15-2020-794).

\section{Конфликт интересов}

Авторы заявляют, что у них нет конфликта интересов.

\section{Список литературы}

[1] А.И. Александров, И.А. Александров, А.И. Прокофьев. Письма в ЖЭТФ, 97 (9), 630 (2013). [A.I. Aleksandrov, I.A. Alexandrov, A.I. Prokof'ev. JETP Lett., $97(9), 546$ (2013). DOI: 10.1134/S0021364013090038]

[2] А.И. Александров, И.А. Александров, А.И. Прокофьев. ДАН сер. физ. хим., 451 (1), 50 (2013). [A.I. Aleksandrov, I.A. Alexandrov, A.I. Prokof'ev. Dokl. Phys. Chem., 451 (1), 147 (2013). DOI:10.1134/S0012501613070014]

[3] А.И. Александров, И.А. Александров, С.Б. Зезин, Е.Н. Дегтярев, А.А. Дубинский, С.С. Абрамчук, А.И. Прокофьев. Химическая физика, 35 (2), 78 (2016). [A.I. Aleksandrov, I.A. Aleksandrov, S.B. Zezin, E.N. Degtyarev, A.A. Dubinskiy, S.S. Abramchuk, A.I. Prokof'ev. Rus. J. Phys. Chem. B, 10 (1), 69 (2016). DOI: 10.1134/S1990793116010139]

[4] А.И. Александров, В.Г. Шевченко, И.А. Александров. Письма в ЖТФ, 46 (7), 43 (2020). DOI: 10.21883/PJTF.2020.07.49220.18119 [A.I. Aleksandrov, V.G. Shevchenko, I.A. Aleksandrov. Tech. Phys. Lett., 46 (4), 346 (2020). DOI: 10.1134/S1063785020040021]

[5] А.И. Александров, В.Г. Шевченко, И.А. Александров, Е.Н. Дегтярев, С.С. Абрамчук. Высокомолек. соед. Сер. А, 62 (5), 380 (2020). [A.I. Aleksandrov, V.G. Shevchenko, I.A. Aleksandrov, E.N. Degtyarev, S.S. Abramchuk. Polymer Sci., Ser. A, 62 (5), 550 (2020). DOI: $10.1134 / \mathrm{S} 0965545 \mathrm{X} 2004001 \mathrm{X}]$ 
[6] R.H. Dicke. Phys. Rev., 93, 99 (1954).

[7] P.W. Bridgman. Rev. Mod. Phys., 18 (1), 1 (1946).

[8] I.J. Dzyaloshinskii. Chem. Sol., 4, 241 (1958).

[9] T. Moriya. Phys. Rev., 120, 91 (1960).

[10] A.I. Aleksandrov, I.A. Aleksandrov, V.G. Shevchenko, A.N. Ozerin. Chinese J. Polym. Sci., 39, 601 (2021). DOI: $10.1007 / \mathrm{s} 10118-021-2511-5$

[11] А.И. Александров, В.Г. Шевченко, И.А. Александров, С.В. Фокин, В.И. Овчаренко. Письма в ЖТФ, 47 (1), 23 (2021). DOI: 10.21883/PJTF.2021.01.50453.18451 [A.I. Alexandrov, V.G. Shevchenko, I.A. Alexandrov, S.V. Fokin, V.I. Ovcharenko, Tech. Phys. Lett., $47(1), 19$ (2021). DOI: 10.1134/S106378502101003X]

[12] Г.В. Романенко, С.В. Фокин, Г.А. Летягин, А.С. Богомяков, В.И. Овчаренко. Журн. структурной химии, 60 (7), 1139 (2019). DOI: 10.26902/JSC_id42975 [G.V. Romanenko, S.V. Fokin, G.A. Letyagin, A.S. Bogomyakov, V.I. Ovcharenko. J. Struct. Chem., 60 (7), 1091 (2019). DOI: $10.1134 / \mathrm{S} 0022476619070102]$

[13] T. Sugawara, H. Komatsu, K. Suzuki. Chem. Soc. Rev., 40, 3105 (2011). DOI: 10.1039/c0cs00157k

[14] I. Barskaya, E. Tretyakov, R. Sagdeev, V. Ovcharenko, E. Bagryanskaya, K. Maryunina, T. Takui, K. Sato, M. Fedin. J. Am. Chem. Soc., 136, 10132 (2014). DOI: $10.1021 / \mathrm{ja} 504774 \mathrm{q}$

[15] S. Nakazawa, S. Nishida, T. Ise, T. Yoshino, N. Mori, R.D. Rahimi, E. Sato, Y. Morita, K. Toyota, D. Shiomi. Angew. Chem. Int. Ed., 51, 9860 (2012). DOI: 10.1063/1.4816636

[16] K. Ayabe, K. Sato, S. Nakazawa, S. Nishida, K. Sugisaki, T. Ise, Y. Morita, K. Toyota, D. Shiomi, M. Kitagawa. Mol. Phys., 111, 2767 (2013). DOI: $10.1080 / 00268976.2013 .811304$

[17] E. Coronado, A.J. Ctein. J. Mater. Chem. 19, 1670 (2009). DOI: $10.1039 / \mathrm{b} 901955 \mathrm{n}$

[18] R. Hussain, S.S. Kruk, C.F. Bonner, M.A. Noginov, I. Staude, Y.S. Kivshar, N. Noginova, D.N. Neshev. Opt. Lett., 40, 8 (2015). DOI: $10.1364 /$ OL.40.001659

[19] Е.Д. Трифонов. ЖЭТФ, 120 (5), 1117 (2001). [E.D. Trifonov. JETP, 93 (5), 969 (2001). DOI:10.1134/1.1427108]

[20] А.В. Андреев, В.И. Емельянов, Ю.А. Ильинский. УФН, 131 (4), 655 (1980). [A.V. Andreev, V.I. Emel'yanov, Yu.A. Il'inskii. Sov. Phys. Usp., 23, 493 (1980). DOI: 10.1070/PU1980v023n08ABEH005024]

[21] В.В. Кочаровский, В.В. Железняков, Е.Р. Кочаровская, В.В. Кочаровский. УФН, 187 (4), 367 (2017). [V.V. Kocharovsky, V.V. Zheleznyakov, E.R. Kocharovskaya, V.V. Kocharovsky. Phys. Usp., 60, 345 (2017). DOI: 10.3367/UFNe.2017.03.038098]

[22] H.A. Lorentz. PhysikalischeZeitschrift, 498, 514 (1899).

[23] T.A. Kaplan, S.D. Mahant. Phys. Rev. B, 83, 174432 (2011). DOI: 10.1103/PhysRevB.83.174432

[24] L. Landau, L. Lifshitz. Physik. Zeitschrift Sowjet Union, 8, 153 (1935).

[25] F. Bloch. Phys. Rev., 70, 460 (1946). DOI:10.1103/PhysRev.70.460

[26] N. Bloembergen. Phys. Rev., 78, 572 (1950). DOI:10.1103/PhysRev.78.572

[27] A.K. Jonscher. Dielectric Relaxation in Solids (Chelsea Dielectric Press Ltd, London, 1983)

[28] А.Г. Гуревич. Ферриты на сверхвысоких частотах (Физматгиз, М., 1960)
[29] S. Havriliak, S. Negami. J. Polym. Sci., 14, 99 (1966). DOI:10.1002/polc.5070140111

[30] S.D. Chemerisov, G.D. Perekhodtsev, D.S. Tipikin, Ya.S. Lebedev, A.I. Prokof'ev, A.I. Aleksandrov, A.A. Dubinskii, K. Mobius, O.G. Poluektov, J. Schmidt. J. Chem. Soc., Faraday Trans., 92 (1 1), 1959 (1996). DOI: $10.1039 / \mathrm{ft} 9969201959$

[31] R.R. Rakhimov, E.M. Jackson, J.S. Hwang, A.I. Prokof'ev, I.A. Alexandrov, A.Y. Karmilov, A.I. Aleksandrov. J. Appl. Phys., 95 (11), 7133 (2004). DOI: 10.1063/1.1668613

[32] А.Л. Бучаченко, В.Л. Бердинский. Успехи химии, 52 (1), 3 (1983). [A.L. Buchachenko, V.L. Berdinskii. Rus. Chem. Bull., 44, 1578 (1995). DOI: 10.1007/BF01151273]

[33] Я.Б. Зельдович, А.Л. Бучаченко, Е.Л. Франкевич. УФН, 155 (1), 3 (1988). [Ya.B. Zel'dovich, A.L. Buchachenko, E.L. Frankevich. Sov. Phys. Usp., 31, 385 (1988). DOI: 10.1070/PU1988v031n05ABEH003544]

[34] А.Л. Бучаченко, В.Л. Бердинский. Изв АН. Сер. хим. 1646 (1995). [A.L. Buchachenko, V.L. Berdinskii. Rus. Chem. Bull., 44, 1578 (1995). DOI:10.1007/BF01151273]

[35] А.Л. Бучаченко, В.Л. Бердинский. Кинетика и катализ, 37 (5), 659 (1996). [V.L. Berdinskii, A.L. Buchachenko. Kinetics and Catalysis, 37, 615 (1996).]

[36] Н.С. Ениколопов. Успехи химии, 60, 586 (1991). [N.S. Enikolopov. Rus. Chem. Rev., 60, 283 (1991). DOI: 10.1070/RC1991v060n03ABEH001062]

[37] С.Н. Журков. ФТТ, 25 (10), 3119 (1983).

[38] E.E. Tomashevskii, V.A. Zakrevskii, I.I. Novak, V.E. Korsukov, V.R. Regel, O.F. Pozdnjakov, A.I. Slutsker, V.S. Kuksenko. Int. J. Fract., 11, 803 (1975). DOI: 10.1007/BF00012898

[39] В.А. Закревский, В.А. Пахотин. ФТТ, 52 (6), 1083 (2010). 